\title{
Extremely Rapid Synthesis of Aliphatic Polyesters by Direct Polycondensation of 1:1 Mixtures of Dicarboxylic Acids and Diols Using Microwaves
}

\author{
Sivan VelmathI,${ }^{\dagger \dagger}$ Ritsuko Nagahata,${ }^{\dagger}$ and Kazuhiko TaKeuChI \\ National Institute of Advanced Industrial Science and Technology (AIST), AIST Tsukuba Central 5, \\ 1-1-1 Higashi, Tsukuba 305-8565, Japan
}

(Received February 28, 2007; Accepted May 16, 2007; Published July 3, 2007)

\begin{abstract}
Direct polycondensation of equimolar quantities of a dicarboxylic acid and a diol, which does not generate any undesirable wastes, is an ideal method for producing aliphatic polyesters, the demand for which is set to increase considerably as a result of their biocompatibility. There is a need for an effective system for producing aliphatic polyesters under mild conditions with short reaction times. We report a rapid, environmentally benign, solventfree method for synthesis of aliphatic polyesters by direct polycondensation of equimolar quantities of a dicarboxylic acid and a diol through microwave irradiation in the presence of tin (II) chloride as a catalyst. [doi:10.1295/polymj.PJ2006248]

KEY WORDS Aliphatic Polyesters / Direct Polycondensation / Solvent-free / Microwaves /
\end{abstract}

Governments in various countries have adopted policy targets that involve making optimal use of "biomass" (organic materials derived from renewable and living organisms as opposed to fossil resources) as a source of energy and raw materials to realize a society that is capable of sustainable development. Resins derived from biomass are called "bio-based plastics", and most such resins produced by chemosynthesis are aliphatic polyesters. Generally, aliphatic polyesters are synthesized by polycondensation of a diol and a dicarboxylic acid as the starting materials. Polycondensation is an equilibrium reaction and, to shift the balance toward the products and to obtain polymers with a high degree of polymerization, eliminated components such as water must be completely removed from the system. Industrially, an excess of the diol or dicarboxylic acid component is used in most cases to compensate for the loss of the lower-boiling component as a result of the depressurized or azeotropic conditions that are required to remove the eliminated components and suppress the reverse reaction.

When materials derived from biomass are used to synthesize polymers by polycondensation, the products are often claimed to be carbon neutral, because the source of the carbon and oxygen in the material is atmospheric $\mathrm{CO}_{2}$, but in reality it is actually difficult to achieve an equimolar reaction and there is a hidden problem in terms of atom economy. Furthermore, a large amount of process energy derived from fossil fuels is still required because of the need for a high reaction temperature and a prolonged reaction time.
In general, a multi-step manufacturing process is adopted in which oligomers with a molecular weight of the order of several thousands are produced initially in a process called "esterification"; these oligomers are subsequently converted into polymers with higher molecular weights. ${ }^{1}$ Since the beginning of the 21 st century, several attempts have been made to synthesize aliphatic polyesters efficiently by an equimolar reaction. These have resulted in dramatic improvements on conventional methods and involve the use of hafnium(IV) or zirconium(IV) salts, ${ }^{2,3}$ rare earth triflates, ${ }^{4,5}$ distannoxane, ${ }^{6}$ or tin(II) chloride ${ }^{7}$ as catalysts. However, the synthetic reactions still require more than $10 \mathrm{~h}$ of reaction time, so there remains a need for a simple but rapid, economical, and environmentally benign manufacturing process.

To solve these problems, we propose a microwave (MW)-assisted polycondensation process in which MW radiation provides the driving force for the reaction. MW-induced reactions form a promising area of study in which many recent examples in organic chemistry and macromolecular chemistry illustrate abilities of MW radiation beyond its simple heating effect, such as rate enhancements and an improvement in selectivity. ${ }^{8}$ We have developed an innovative technique for the production of condensation polymers by focusing on the fact that polycondensation is a reaction mainly involving substrates and by eliminating components that efficiently absorb MW radiation. ${ }^{9,10}$ Here, we report a highly efficient and direct polycondensation of stoichiometric amounts of a dicarboxylic acid and a diol by using MW irradiation in the pres-

\footnotetext{
${ }^{\dagger}$ To whom correspondence should be addressed (Tel: +81-29-861-6325, Fax: +81-29-861-6327, E-mail: nagahata-ritsuko@aist.go.jp).

${ }^{\dagger \dagger}$ Present address: Department of Chemistry, National Institute of Technology, Tiruchirappalli-620 015, India
} 


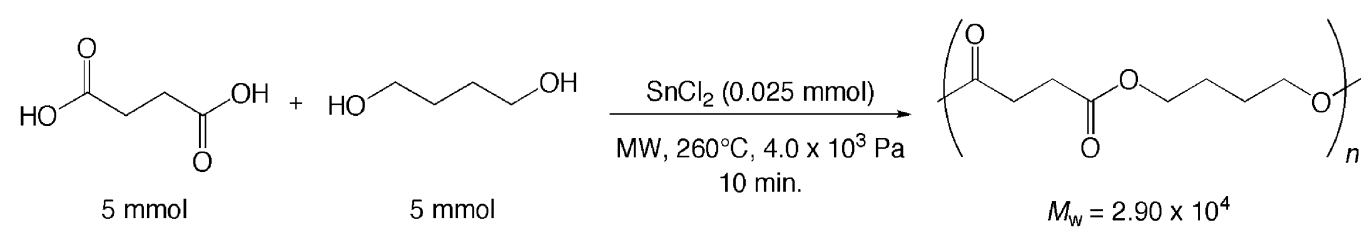

Scheme 1.

ence of $\mathrm{SnCl}_{2}$ as a catalyst. Green catalysts not only require a high catalytic activity and atom efficiency, but also a low toxicity, a low cost, and an ease of handling. $\mathrm{SnCl}_{2}$ is cheaper than the other metal salts and is not considered to be particularly poisonous (rat oral $\mathrm{LD}_{50}: 700 \mathrm{mg} / \mathrm{kg}$.) In the present study, poly(butylene succinate) (PBS) was obtained with a weight-average molecular weight $\left(M_{\mathrm{w}}\right)$ of $2.90 \times 10^{4}$ in the incredibly short time of $10 \mathrm{~min}$. The effects of various reaction parameters, such as the catalyst concentration, MW irradiation time, and temperature, in the syntheses of some aliphatic polyesters are described in detail.

\section{EXPERIMENTAL}

\section{Materials}

Succinic acid and sebacic acid were purchased from Wako Pure Chemical Industries, Ltd. and purified by recrystallization from acetone. 1,4-butanediol (Wako Pure Chemical Industries, Ltd.) were distilled under reduced pressure. Reagent grade tin(II) chloride $\left(\mathrm{SnCl}_{2}\right)$ (Wako Pure Chemical Industries, Ltd.) and all the other reagents were used as received. Analytical grade chloroform and methanol were used as received.

\section{Synthesis of Poly(butylene succinate) (PBS)}

Typically, 1,4-butanediol ( $5 \mathrm{mmol}, 0.450 \mathrm{~g}$ ), succinic acid (5 mmol, $0.590 \mathrm{~g}$ ), and the catalyst $\mathrm{SnCl}_{2}$ $(0.025 \mathrm{mmol}, 0.0047 \mathrm{~g})$ were placed in a flask and kept in the CEM focused MW synthesis system (model Discover). The mixture was stirred at $260^{\circ} \mathrm{C}$ for the required time under $4.0 \times 10^{3} \mathrm{~Pa}$ at maximum $\mathrm{MW}$ power of $200 \mathrm{~W}$. (Note: Since, the system was set at constant temperature mode; MW power was not always $200 \mathrm{~W}$.) Then the reaction mixture was cooled to room temperature. The polymer formed was dissolved in chloroform $(20 \mathrm{~mL})$ and precipitated in methanol $(200 \mathrm{~mL})$.

For comparison polymerization under conventional heating was carried out in an oil bath preheated to $260^{\circ} \mathrm{C}$. Equimolar quantities of monomers and 0.5 mol \% of catalyst were placed in a round-bottomed flask fitted with a condenser and a three way take off adapter (for nitrogen inlet and outlet) connected to the bubbler. The reaction mixture was heated at $260^{\circ} \mathrm{C}$ for ten minutes. After the completion of reac- tion, reaction mixture was cooled and the polymer formed was dissolved in choloroform and precipitated with methanol.

\section{Analytical Measurements}

The polymer formed was characterized using NMR, IR, and SEC analyses. ${ }^{1} \mathrm{H}$ and ${ }^{13} \mathrm{C}$ NMR were recorded on a JEOL LA600 in $\mathrm{CDCl}_{3}$ with tetramethylsilane as internal standard. FT-IR spectra were measured on a Perkin-Elmer FT-IR spectrometer as $\mathrm{KBr}$ plates. SEC measurements were performed with a JASCO GULLIVER system coupled with a Shodex K-407L column and an RI detector. The mobile phase was $\mathrm{CHCl}_{3}$ and the data were recorded at the flow rate of $0.3 \mathrm{~mL} /$ minute. Average molecular weights of the polymerization products were determined with polystyrenes as standard.

\section{RESULTS AND DISCUSSION}

We first investigated the polymerization of succinic acid and 1,4-butanediol under MW irradiation (Scheme 1). An efficient direct polycondensation process requires two factors: effective removal of water, and suppression of depolymerization. Polyesterification is a reversible reaction and the rate of the reverse reaction increases if water is not efficiently removed from the reaction mixture. The usual route adopted for efficient removal of water is azeotropic removal with $o$-xylene or toluene; however, the slight solubility of water in these hydrocarbons hinders the effective removal of water. Thus in the present study, polyesterification was carried out by the bulk polymerization method (because the best solvent is no solvent) under a vacuum of $4.0 \times 10^{3} \mathrm{~Pa}$ for effective removal of the water eliminated during the reaction. For a start, the polyesterification was carried out by bulk polymerization method, with catalyst concentration of $2 \mathrm{~mol} \%$, reaction temperature of $220^{\circ} \mathrm{C}$, stoichiometry of 1:1.2 of dicarboxylic acid and diol respectively and, MW irradiation time of 20 minutes. The catalysts chosen were well known polyesterification catalysts such as $\mathrm{Ti}(\mathrm{Oipr})_{4}, \mathrm{ZrCl}_{4}, \mathrm{SnCl}_{2}, p$-TSA. $\mathrm{H}_{2} \mathrm{O}$, $\mathrm{HfCl}_{4} .2 \mathrm{THF}, \mathrm{Sc}(\mathrm{Otf})_{3}$, and Dibutyldichlorotin(IV). The results obtained are given in Figure 1.

Both $\mathrm{SnCl}_{2}$ and $p$-TSA. $\mathrm{H}_{2} \mathrm{O}$ were found to be effective catalysts under present condition. Ti(Oipr $)_{4}$ and 


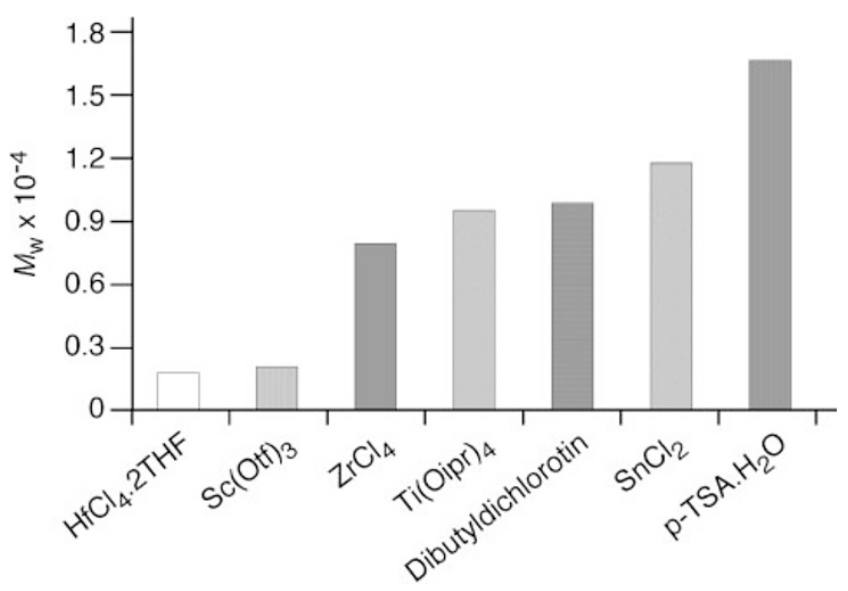

Figure 1. Microwave-assisted synthesis of PBS using different catalysts.

$\mathrm{ZrCl}_{4}$ were found to be moderately effective. However, $\mathrm{HfCl}_{4}$. $2 \mathrm{THF}$ and $\mathrm{Sc}(\mathrm{Otf})_{3}$ which are well known for the synthesis of PBS were either less active or inert under MW irradiation condition. To optimize the reaction condition to achieve high molecular weight, polymerization reaction was explored in detail with $\mathrm{SnCl}_{2}$ catalyst. We have optimised the conditions for $p$-TSA. $\mathrm{H}_{2} \mathrm{O}$, those results will be published elsewhere. The recent concern towards environmental problems prompted us to explore the most ideal polycondensation route, which involves stoichiometric quantities of monomers, no waste (except water as the only byproduct), and solvent free conditions. PBS synthesis was carried out with equivalents of succinic acid and butanediol. To start with the polycondensation reaction was studied at various temperatures from 220 to $260^{\circ} \mathrm{C}$. The reaction time was fixed at $10 \mathrm{~min}$ at a catalyst concentration of $0.5 \mathrm{~mol} \%$ : the results are shown in Table I. The molecular weight of the polymer obtained increased with increasing reaction temperature. For each $20^{\circ} \mathrm{C}$ rise in temperature, the $M_{\mathrm{w}}$ of the polymer doubled (runs $1-3$ ). At $260^{\circ} \mathrm{C}$, the $M_{\mathrm{w}}$ of PBS was $2.90 \times 10^{4}$ and the isolated yield was $88.1 \%$ (run 3 ). The reaction was quantitative; no monomers were isolated from the reaction mixture. The polyester that was obtained was white in color. The maximum reaction temperature was limited to $260^{\circ} \mathrm{C}$ to avoid any discoloration of the resulting polyester. Next, the effect of the MW irradiation time on the molecular weight of the PBS formed was studied by varying the MW irradiation time (10, 20, 30, and $40 \mathrm{~min}$ ) while keeping the other parameters constant (runs 3-6). It is evident from the table that an excessive increase in the reaction time resulted in a decrease in the molecular weight of the PBS formed. This may be due to degradation of the polymer as a result of prolonged MW irradiation. Finally, the role of catalyst concentration was studied by varying the
Table I. Direct esterification of dicarboxylic acids with diols catalyzed by $\mathrm{SnCl}_{2}$. Unless otherwise noted, the monomers

( $5 \mathrm{mmol}$ of each) and $\mathrm{SnCl}_{2}$ catalyst were subjected to MW irradiation at a pressure of $4.0 \times 10^{3} \mathrm{~Pa}$

\begin{tabular}{cccccc}
\hline Run & $\begin{array}{c}\text { Cat. Conc. } \\
(\mathrm{mol} \%)\end{array}$ & $\begin{array}{c}\text { Temp. } \\
\left({ }^{\circ} \mathrm{C}\right)\end{array}$ & $\begin{array}{c}\text { Time } \\
(\mathrm{min})\end{array}$ & $M_{\mathrm{n}}{ }^{\mathrm{a}} \times 10^{-4}$ & $M_{\mathrm{w}}{ }^{\mathrm{a}} \times 10^{-4}$ \\
\hline 1 & 0.5 & 220 & 10 & 0.19 & 0.43 \\
2 & 0.5 & 240 & 10 & 0.99 & 2.05 \\
3 & 0.5 & 260 & 10 & 1.40 & 2.90 \\
4 & 0.5 & 260 & 20 & 0.93 & 2.30 \\
5 & 0.5 & 260 & 30 & 1.13 & 2.25 \\
6 & 0.5 & 260 & 40 & 1.12 & 1.74 \\
7 & 0 & 260 & 10 & $-^{\mathrm{b}}$ & $-^{\mathrm{b}}$ \\
8 & 0.1 & 260 & 10 & 0.37 & 0.72 \\
9 & 1.0 & 260 & 10 & 0.80 & 1.75 \\
$10^{\mathrm{c}}$ & 0.5 & 260 & 10 & 0.23 & 0.43 \\
$11^{\mathrm{d}}$ & 0.5 & 260 & 10 & 0.12 & 0.20 \\
\hline
\end{tabular}

${ }^{a}$ Measured by $\mathrm{SEC}$ in $\mathrm{CHCl}_{3}$, based on standard polystyrene. ${ }^{b} \mathrm{No}$ polymer was precipitated in $\mathrm{MeOH}$. ${ }^{\mathrm{c}} \mathrm{No}$ vacuum. ${ }^{\mathrm{d}}$ No MW irradiation (the reaction was conducted in a preheated oil bath).

catalyst concentration from 0 to $1.0 \mathrm{~mol} \%$, keeping the other parameters constant (runs 3, 7-9). In the absence of $\mathrm{SnCl}_{2}$, no polymer was isolated indicating that a catalyst is necessary for the polymerization reaction to proceed (run 7). PBS with the highest molecular weight was obtained at a $\mathrm{SnCl}_{2}$ concentration of $0.5 \mathrm{~mol} \%$ (run 3). Under atmospheric pressure the reaction resulted in oligomers only (run 10); this may be due to the lack of removal of water eliminated from the reaction medium, and thus the predomination of the reverse reaction.

MW-assisted chemical reactions have been studied with great interest for the past three decades. Since then there has been considerable debate over the true rate enhancement mechanism of $\mathrm{MW}$-assisted reactions as rapid increase in reaction temperature could result in significant rate enhancement. Recently Jachuck et al. ${ }^{11}$ investigated the influence of MW irradiation in the benzyl alcohol oxidation reaction under isothermal conditions and found that MW does invigorate the molecules to achieve higher reaction rate. In order to prove the efficiency of the MW-assisted polymerization reactions, PBS synthesis was studied using $\mathrm{SnCl}_{2}$ catalysts under conventional oil bath heating. All reaction conditions were set identical except for the heating mode. The molecular weight of the polymer formed after $10 \mathrm{~min}$. heating was very low in the absence of MW irradiation (run 11). Since MW radiation is not sufficiently energetic to cause either ionization or electronically excited state chemistry to occur, the overall reaction mechanism may be similar to that of thermal reaction. But the drastic increase in the reaction rate in the case of $\mathrm{MW}$-assisted reaction 
Table II. Synthesis of aliphatic polyesters using $\mathrm{SnCl}_{2}$ catalyst and MW. Reaction conditions: $5 \mathrm{mmol}$ dicarboxylic acid, $5 \mathrm{mmol}$ of diol, and $0.025 \mathrm{mmol}$ of $\mathrm{SnCl}_{2}$ were kept under MW irradiation for 10 minutes at $260^{\circ} \mathrm{C}$ under a pressure of $4.0 \times 10^{3} \mathrm{~Pa}$

\begin{tabular}{lc}
\hline Polyester & $M_{\mathrm{w}}{ }^{\mathrm{a}} \times 10^{-4}$ \\
\hline Poly(ethylene succinate) & 1.90 \\
Poly(propylene succinate) & 1.22 \\
Poly(butylene succinate) & 2.90 \\
Poly(1,4-cyclohexanedimethylene succinate) & 3.60 \\
Poly(ethylene sebacate) & 0.51 \\
Poly(propylene sebacate) & 1.04 \\
Poly(butylene sebacate) & 0.99 \\
Poly(1,4-cyclohexanedimethylene sebacate) & 1.03 \\
\hline
\end{tabular}

${ }^{\mathrm{a}}$ Measured by $\mathrm{SEC}$ in $\mathrm{CHCl}_{3}$, based on standard polystyrene.

might be attributed to the effective interaction of the MW irradiation with the water eliminated in the reaction resulting in their removal from the reaction system and thus driving the reaction equilibrium in the forward direction.

The newly developed MW-assisted synthesis helps to achieve the synthesis of PBS with a molecular weight of several tens of thousands in a rapid and environmentally friendly way in the incredibly short reaction time of $10 \mathrm{~min}$. Polymers of such high molecular weights can be easily pelletized and can thus be processed by post-polymerization to form high-molecular weight polymers suitable for practical applications, as has already been established as an industrial method.

To explore the generality and scope of the $\mathrm{SnCl}_{2}$ catalyzed polyesterification, we extended the scope of the reaction to the synthesis of various other aliphatic polyesters. The results are given in Table II. In most cases, the polycondensation proceeded quantitatively; however, with sebacic acid, the $M_{\mathrm{w}}$ of the polymer obtained was slightly lower than that of succinic acid-based polymers. It is known that the molecular weight of polyesters depends on the chain lengths of the dicarboxylic acid and the diol components.

\section{CONCLUSIONS}

We succeeded in synthesizing aliphatic polyesters by direct polyesterification of a dicarboxylic acid with a diol using $\mathrm{SnCl}_{2}$ as a catalyst under MW irradiation.
The molecular weight of the polymers that were obtained was relatively constant and the reaction time was extremely short $(10 \mathrm{~min})$ compared with existing systems, such as $\mathrm{Sc}(\mathrm{OTf})_{3}\left(M_{\mathrm{n}}=2.09 \times 10^{4}, 4.0 \times\right.$ $\left.10^{3} \mathrm{~Pa}, 10 \mathrm{~h}\right),{ }^{5} \mathrm{HfCl}_{4}(\mathrm{THF})_{2},\left(M_{\mathrm{w}}=3.40 \times 10^{4}, 24 \mathrm{~h}\right.$, $o$-xylene reflux $),{ }^{2} \mathrm{SnCl}_{2}\left(M_{\mathrm{n}}=1.64 \times 10^{4}, 16 \mathrm{~h}\right.$, dimethyl benzene reflux). ${ }^{7}$ The advantages of this method, such as equimolar direct polycondensation, a short reaction time, and solvent-free conditions, permit the synthesis of aliphatic polyesters in a rapid and ecofriendly manner under mild conditions that are mild compared with those used in conventional syntheses. The new procedure will make the road to green polymer chemistry more accessible. The synthetic ability of organic chemists coupled with the wealth of MW fabrication techniques that are available to engineers will contribute to the realization of the commercialization of the MW-assisted polymerization method to meet our future needs.

Acknowledgment. A part of this work was financially supported by the Industrial Technology Research Grant Program in 2006 from the New Energy and Industrial Technology Development Organization (NEDO) of Japan.

\section{REFERENCES}

1. E. Takiyama, I. Niikura, and Y. Hatano, U. S. Patent 5306 787 (1994).

2. K. Ishihara, M. Nakayama, S. Ohara, and H. Yamamoto, Tetrahedron, 58, 8179 (2002).

3. K. Ishihara, S. Ohara, and H. Yamamoto, Science, 290, 1140 (2000).

4. A. Takasu, Y. Iio, Y. Oishi, Y. Narukawa, and T. Hirabayashi, Macromolecules, 38, 1048 (2005).

5. A. Takasu, Y. Oishi, Y. Iio, Y. Inai, and T. Hirabayashi, Macromolecules, 36, 1772 (2003).

6. M. Ishii, M. Okazaki, Y. Shibasaki, M. Ueda, and T. Teranishi, Biomacromolecules, 2, 1267 (2001).

7. C. Zhu, Z. Zhang, Q. Liu, Z. Wang, and J. Jin, J. Appl. Polym. Sci., 90, 982 (2003).

8. C. O. Kappe, Angew. Chem. Int. Ed., 43, 6250 (2004).

9. R. Nagahata, J. Sugiyama, S. Velmathi, Y. Nakao, M. Goto, and Takeuchi, Polym. J., 36, 483 (2004).

10. S. Velmathi, R. Nagahata, J. Sugiyama, and K. Takeuchi, Macromol. Rapid. Commun., 26, 1163 (2005).

11. R. J. J. Jachuck, D. K. Selvaraj, and R. S. Varma, Green Chem., 8, 29 (2006). 